\title{
Kinetic Modelling of Starch and Lipid Formation during Mixotrophic, Nutrient-limited Microalgal Growth
}

DOI:

10.1016/j.biortech.2017.05.177

\section{Document Version}

Accepted author manuscript

Link to publication record in Manchester Research Explorer

\section{Citation for published version (APA):}

Figueroa Torres, G., Pittman, J., \& Theodoropoulos, C. (2017). Kinetic Modelling of Starch and Lipid Formation during Mixotrophic, Nutrient-limited Microalgal Growth. Bioresource Technology, 241.

https://doi.org/10.1016/j.biortech.2017.05.177

\section{Published in:}

Bioresource Technology

\section{Citing this paper}

Please note that where the full-text provided on Manchester Research Explorer is the Author Accepted Manuscript or Proof version this may differ from the final Published version. If citing, it is advised that you check and use the publisher's definitive version.

\section{General rights}

Copyright and moral rights for the publications made accessible in the Research Explorer are retained by the authors and/or other copyright owners and it is a condition of accessing publications that users recognise and abide by the legal requirements associated with these rights.

\section{Takedown policy}

If you believe that this document breaches copyright please refer to the University of Manchester's Takedown Procedures [http://man.ac.uk/04Y6Bo] or contact uml.scholarlycommunications@manchester.ac.uk providing relevant details, so we can investigate your claim.

\section{OPEN ACCESS}


1 Kinetic Modelling of Starch and Lipid Formation during Mixotrophic,

\title{
Nutrient-limited Microalgal Growth
}

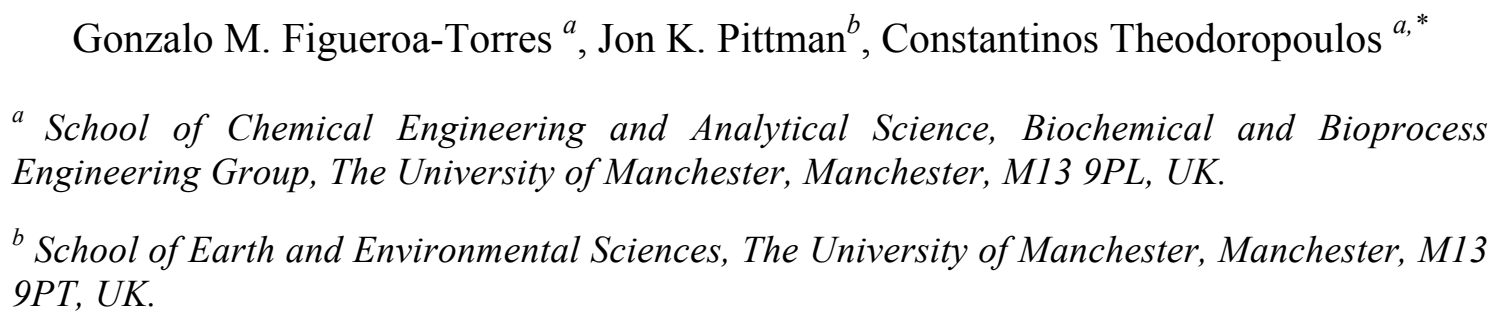

\begin{abstract}
Microalgal starch and lipids, carbon-based storage molecules, are useful as potential biofuel feedstocks. In this work, cultivation strategies maximising starch and lipid formation were established by developing a multi-parameter kinetic model describing microalgal growth as well as starch and lipid formation, in conjunction with laboratoryscale experiments. Growth dynamics are driven by nitrogen-limited mixotrophic conditions, known to increase cellular starch and lipid contents whilst enhancing biomass growth. Model parameters were computed by fitting model outputs to a range of experimental datasets from batch cultures of Chlamydomonas reinhardtii. Predictive capabilities of the model were established against different experimental data. The model was subsequently used to compute optimal nutrient-based cultivation strategies in terms of initial nitrogen and carbon concentrations. Model-based optimal strategies yielded a significant increase of $261 \%$ for starch $\left(0.065 \mathrm{gC} \mathrm{L}^{-1}\right)$ and $66 \%$ for lipid $(0.08$ $\left.\mathrm{gC} \mathrm{L}^{-1}\right)$ production compared to base-case conditions $\left(0.018 \mathrm{gC} \mathrm{L}^{-1}\right.$ starch, $0.048 \mathrm{gC} \mathrm{L}^{-1}$ lipids).
\end{abstract}

Keywords: Biofuels, microalgal dynamics, kinetic modelling, starch and lipids optimisation, Chlamydomonas reinhardtii 


\section{Introduction.}

Our current dependence on fossil fuels raises two major concerns: the overexploitation of finite crude oil resources and the associated emissions of greenhouse gases (GHG) leading to global warming (Scaife et al., 2015). About $60 \%$ of the fossil fuels directed annually towards primary energy consumption are taken up by the transportation sector (Escobar et al., 2009). Although biofuels have emerged as a suitable and renewable replacement for transport-associated fuels such as gasoline and diesel, sustainable and cost-effective biofuel production systems must first be developed (Escobar et al., 2009; Scaife et al., 2015).

Due to exhibiting faster growth rates than terrestrial plants, microalgal biomass has recently been considered as a potential biofuel feedstock (Brennan and Owende, 2010), as opposed to traditional food-based or lignocellulosic substrates which compete for food or arable land (Scaife et al., 2015). Microalgae are photosynthetic organisms which synthesize biologically important compounds such as carbohydrates, lipids, proteins and nucleic acids, acting as storage or functional elements (Brennan and Owende, 2010; Choix et al., 2012). In particular, their internal pool of carbohydrates and lipids has directed the attention towards the use of microalgae as a renewable feedstock for sugar and lipid-based fuels.

In green microalgae, the main storage carbohydrate synthesized by cells is starch, which is located within the chloroplast in the form of granules (Choix et al., 2012; Markou et al., 2012). Meanwhile, oil bodies are found in the cytosol and chloroplast (Ball and Deschamps, 2009; Goodson et al., 2011). Cellular contents of starch and lipids (triacylglycerol, TAG) have been shown to increase under nitrogen-starved conditions (Bajhaiya et al., 2016). Nevertheless, enhanced starch and lipid accumulation rates 
51 come hand-in-hand with a decrease in biomass growth (Markou et al., 2012), suggesting

52 the need to identify an optimal balance between microalgal growth and starch and lipid

53 accumulation.

54 Microalgal cells can be photototrophic or heterotrophic depending on whether their carbon fixation route requires the presence of an inorganic or an organic carbon source (Brennan and Owende, 2010). Some strains are able to grow mixotrophically by

57 utilizing both inorganic and organic carbon sources. In most cases, mixotrophic

58 cultivation leads to improved growth rates against standard phototrophic conditions

59 (Chapman et al., 2015; Johnson and Alric, 2013), causing substantial increases in biomass productivities (Moon et al., 2013). Consequently, quantifying starch and lipid accumulation during nitrogen-limited mixotrophic growth is of great relevance for microalgae-based biofuels.

Chlamydomonas reinhardtii, the chosen microalgal strain in this work, has been widely studied (e.g. Goodenough et al., 2014; Bajhaiya et al., 2016). Its carbon metabolism is well known (Johnson and Alric, 2013), making it suitable for the analysis of the carbon assimilation and its distribution between starch and lipid reserves. In particular, a clear increase in starch and lipid accumulation for mixotrophic growth under nutrient stress has been observed for this strain (Bajhaiya et al., 2016; Bekirogullari et al., 2017). Nevertheless, despite nutrient stress being regarded as a simple and cost-effective strategy to enhance starch and lipid formation, optimisation of the process is required for viable large-scale cultivation.

Robust kinetic models capable of simultaneously predicting starch and lipid formation can significantly aid in the establishment of optimal cultivation strategies. Models that 
take into account the structured and segregated (i.e. each cell behaves as an individual unit with dynamic composition) nature of cells, and/or even the stochastic nature of cell growth (Alonso et al., 2014) can realistically predict the formation of multiple intracellular components as well as cells' response to cultivation conditions. Such models can provide useful insights about the algal metabolic networks and intracellular fluxes (Chapman et al., 2015; Rügen et al., 2012), but are usually highly complex and computationally expensive (Shuler and Kargi, 1992). Unstructured non-segregated models, on the other hand, have a simpler formulation (assume all cells in culture are identical), but have been shown to be applicable to practical algal cultivation systems.

Most of the existing unstructured models for microalgae, however, have focused solely on the simulation of lipid production (Bekirogullari et al., 2017; Packer et al., 2011). These models assume that lipid formation is a consequence of excess carbon (between the amount fixated and the amount required for cell growth) directed towards synthesis of lipids rather than other carbon-based elements, such as cellular organelles or proteins. Although this assumption is in agreement with the carbon pathways of microalgae, any excess of assimilated carbon is also directed towards formation of starch reserves (Johnson and Alric, 2013). Models for nutrient-limited algal growth considering both sugar and lipids dynamics have been proposed by Mairet et al., 2011b and Kumar et al., 2016, but with a focus on lipid production during phototrophic growth. Mixotrophic dynamics were proposed by Adesanya et al., 2014, but encompassing sugar and lipids into one single storage molecule, preventing the identification of each individual profile. Thus, the aim of this work is to develop a predictive multi-parameter model for the simultaneous optimization of starch and lipid formation during nitrogen-limited mixotrophic microalgal growth. Our proposed model couples both carbon (C) and 
nitrogen $(\mathrm{N})$ substrates. The model is fitted and validated against datasets obtained from lab-scale culture experiments under different $\mathrm{N}$ and $\mathrm{C}$ regimes. The validated model can then be used with confidence for the identification of optimal cultivation conditions for maximum starch and lipid production.

\section{Materials and Methods.}

\subsection{Strain and cultivation.}

All experiments were carried out with the wild-type strain C. reinhardtii CCAP11/32C, obtained from the Culture Collection of Algae and Protozoa, UK. The strain was maintained under batch mixotrophic conditions in Tris-Acetate-Phosphate (TAP) medium (Harris, 1989) at a temperature of $25^{\circ} \mathrm{C}$. Prior to lab-scale experimentation, an initial algal inoculum was propagated in $150 \mathrm{~mL}$ of TAP medium up to the late exponential phase (5-7 days). This inoculum was placed in an orbital shaker at $150 \mathrm{rpm}$ and constant illumination of $125 \mu \mathrm{mol} \mathrm{m} \mathrm{m}^{-2}$ (from above) in a light/dark cycle of 16/8 hours. All further lab-scale experimental tests were carried out at the same environmental growth conditions in vessels containing $500 \mathrm{~mL}$ of sterile culture medium and $1 \mathrm{~mL}$ of algal inoculum.

\subsection{Lab-scale culture experiments.}

In order to evaluate nitrogen and carbon effects on microalgal growth as well as starch and lipid accumulation, lab-scale cultures of $C$. reinhardtii were grown under different nitrogen and acetate concentrations. Given that TAP medium is the most routinely used growth medium for this strain and allows results to be compared with other studies, a "control" culture was grown in TAP medium, in which $N_{o}=0.3824 \mathrm{gN} \mathrm{L}^{-1}$ and $A_{o}=$ $0.42 \mathrm{gC} \mathrm{L}^{-1}$. Subsequently, and whilst keeping constant the concentration of all the 
remaining TAP components, acetate-dependent cultures were grown in: $A_{0}=0.21 \mathrm{gC} \mathrm{L}^{-}$

$122{ }^{1}(\mathrm{~A}-), 0.75 \mathrm{gC} \mathrm{L}^{-1}(\mathrm{~A}+), 1.26 \mathrm{gC} \mathrm{L}^{-1}(\mathrm{~A}++)$, and $2.52 \mathrm{gC} \mathrm{L}^{-1}$ (High A). Similarly,

123 nitrogen-dependent cultures were grown in: $N_{0}=0.3350 \mathrm{gN} \mathrm{L}^{-1}(\mathrm{~N}--), 0.3568 \mathrm{gN} \mathrm{L}^{-1}$

$124(\mathrm{~N}-)$, and $0.7430 \mathrm{gN} \mathrm{L}^{-1}($ High $\mathrm{N})$. Two additional cultures were grown by

125 simultaneously changing the initial concentrations of nitrogen and acetate: one in 2.52

$126 \mathrm{gC} \mathrm{L}^{-1}$ and $0.7430 \mathrm{gN} \mathrm{L}^{-1}$ (High A-N), and another in $1.16 \mathrm{gC} \mathrm{L}^{-1}$ and $0.3151 \mathrm{gN} \mathrm{L}^{-1}\left(\mathrm{~A}^{\prime}-\right.$

127 N'). Samples were taken daily during the cultivation period, until cells attained the stationary phase after 8 days. Sufficient identical culture vessels were prepared to allow for duplicate samples to be fully harvested (sacrificed) at each sampling time. For the nitrogen-dependent cultures, media was prepared by modifying exclusively the initial concentration of ammonium chloride $\left(\mathrm{NH}_{4} \mathrm{Cl}\right)$ in the TAP medium, which contains two other nitrogen sources: i) Tris-base buffer $\left(\mathrm{H}_{2} \mathrm{NC}\left(\mathrm{CH}_{2} \mathrm{OH}\right)_{3}\right)$, and ii) ammonium molybdate tetrahydrate, a smaller trace element $\left(\left(\mathrm{NH}_{4}\right)_{6} \mathrm{Mo}_{7} \mathrm{O}_{24} \cdot 4 \mathrm{H}_{2} \mathrm{O}\right)$. The total concentration of these two nitrogen sources amounted to $0.2844 \mathrm{gN} \mathrm{L}^{-1}$ and was kept

Microalgal growth was measured in terms of dry cell weight (DCW), quantified by harvesting all $500 \mathrm{~mL}$ cultures for $3.5 \mathrm{~min}$ at 3,000 $\mathrm{g}$ in an Eppendorf Centrifuge 5424. 
$70^{\circ} \mathrm{C}$. The DCW was then measured gravimetrically in an analytical balance (Sartorius M-Pact AX124, Germany). Samples of the supernatant were stored in $50 \mathrm{~mL}$ Falcon tubes and frozen at $-20^{\circ} \mathrm{C}$ for further analysis of the acetate and nitrogen concentrations.

\subsubsection{Starch and lipid quantification.}

The starch content of cells was quantified according to a Total Starch Assay kit (Megazyme International, Ireland). Briefly, this assay consists of high temperature, two-stage ( $\alpha$-amylase and $\beta$-amyloglucosidase) enzymatic hydrolysis which solubilises starch and releases free D-glucose. The concentration of free D-glucose was determined colourimetrically by measuring sample absorbance values at $508 \mathrm{~nm}$ against a Dglucose standard curve. Total starch concentration was then calculated by multiplying D-glucose concentration by $0.9(162 / 180$, a factor adjusting free D-glucose to anhydrous D-glucose). Quantification of the lipid content was determined by solvent extraction in a SOXTEC Unit 1043 over a triple-stage procedure involving: extraction, rinsing, and solvent recovery (Bekirogullari et al., 2017). Hexane (ACS spectrophotometric grade, $\geq 98.5 \%$, Sigma Aldrich, UK) was used as extracting solvent since it has shown to perform well as an extracting agent of neutral lipids (TAGs) induced under nitrogen-deprived conditions (McNichol et al., 2012). Prior to extraction, dried cell pellets were pulverised by a double-cycle of liquid nitrogen immersion and manual grinding with mortar and pestle. Pulverized cells were then placed in cellulose extraction thimbles ( $26 \times 60 \mathrm{~mm}$, thickness $1.5 \mathrm{~mm}$, Whatman, UK) and positioned in the SOXTEC unit. Extracted lipids were then measured gravimetrically. Starch and lipid concentration is reported in volumetric terms $\left(\mathrm{g} \mathrm{L}^{-1}\right)$, calculated by relating the storage content $(\%)$ of each sample with the corresponding total DCW medium concentration. 


\subsubsection{Acetate concentration.}

169 The residual acetate concentration was measured by High Pressure Liquid

170 Chromatography (HPLC) in a Hi-Plex $8 \mu \mathrm{m} 300 \times 7.7 \mathrm{~mm}$ column using sulphuric acid

$171\left(\mathrm{H}_{2} \mathrm{SO}_{4}\right) 5 \mathrm{mM}$ as mobile phase at a flow rate of $0.6 \mathrm{~mL} \mathrm{~min}^{-1}$ and a temperature of 50

${ }^{\circ} \mathrm{C}$. Acetate was identified by a UV detector at a wavelength of $210 \mathrm{~nm}$. Prior to analysis, all supernatant and calibration samples were filtered in $0.45 \mu \mathrm{m}$ nitrocellulose membranes (Millipore Ltd.) and diluted appropriately in HPLC grade water.

\subsubsection{Total nitrogen and nitrogen quota.}

The residual concentration of total nitrogen was measured in a Total Organic nitrogen source. All experimental samples were diluted appropriately in distilled water. The nitrogen quota, $q_{N}$, at each sampling time point was calculated according to Eq. (1), which is equivalent to the one employed by Bougaran, Bernard, \& Sciandra (2010) to quantify phosphorus cell quotas:

$$
q_{N}=\frac{N_{o}-N}{X}
$$

183 Here $N_{o}$ is the initial concentration of total nitrogen in the medium, and $N$ and $X$ are the

184 residual concentrations of total nitrogen and biomass (DCW), respectively.

\subsubsection{Active biomass and carbon equivalent concentrations.} subtracting the concentration of storage molecules from the total biomass (DCW) 
concentration. The elemental composition of the active biomass fraction was assumed

to be constant in all experiments regardless of the nutrient regime in which the cultures were grown, taken as $\mathrm{CH}_{1.75} \mathrm{O}_{0.56} \mathrm{~N}_{0.08}$, reported for $C$. reinhardtii by Eriksen et al. (2007). In all computations, the concentration of each carbon-based compound was expressed in terms of their specific carbon content, for which the following conversion factors were employed $\left(\mathrm{gC} \mathrm{g}^{-1}\right)$ : 0.444 for starch, 0.77 for lipids $\left(\mathrm{C}_{55} \mathrm{H}_{98} \mathrm{O}_{6}\right), 0.40$ for acetate, and 0.504 for the active biomass fraction.

\section{Model construction.}

A multi-parameter kinetic model was developed to predict $C$. reinhardtii growth and formation of starch and lipid under mixotrophic conditions. The model includes 8 state variables: total biomass $\left(X, \mathrm{gC} \mathrm{L}^{-1}\right)$, total nitrogen $\left(N, \mathrm{gN} \mathrm{L}^{1}\right)$, nitrogen quota $\left(q_{N}, \mathrm{gN}\right.$ $\left.\mathrm{gC}^{-1}\right)$, acetate $\left(A, \mathrm{gC} \mathrm{L}^{-1}\right), \operatorname{starch}\left(S, \mathrm{gC} \mathrm{L}^{-1}\right)$, lipids $\left(L, \mathrm{gC} \mathrm{L}^{-1}\right)$, active biomass $\left(x^{*}, \mathrm{gC} \mathrm{L}^{-}\right.$ ${ }^{1}$ ), and $\mathrm{pH}(H)$. Total biomass is equivalent to the sum of the two major carbon-based compartments: the storage pool made up of starch and lipids, and the active biomass. Microalgal growth and the formation of each cellular component are regulated by the flows shown in Figure 6.

Carbon flows were based on those presented by Mairet et al. (2011) for the microalgal strain Isochrysis aff. Galbana, in which the carbon source was assumed to be directed initially towards sugar synthesis. In the present model carbon assimilation is initially directed towards the formation of active biomass so as to follow more closely the central carbon metabolism of $C$. reinhardtii. A detailed diagrammatic representation of this metabolism is provided by Johnson and Alric (2013), which shows that assimilation of acetate is not only used for starch formation, but also for other important functions 
such as cellular respiration, flagellar motion, and formation of acetyl-CoA, a precursor of numerous biochemical reactions.

213

214

215

216

The cellular flows for carbon assimilation as well as for nitrogen uptake, as depicted in

Figure 6, are regulated by six governing equations: the specific growth rate, $\mu$, the nitrogen uptake rate, $\rho_{N}$, and the intracellular reaction rates $R_{1}, R_{2}, R_{3}$, and $R_{4}$. These equations are described below. All definitions and corresponding units of the kinetic parameters used in the model are listed in Table 1.

\subsection{Specific growth rate, $\mu$}

One of the most widespread equations for microalgal growth is the Droop model (Eq.(2)), where the growth rate, $\mu$, is linked to the internal quota, $q$, of a limiting nutrient ( $q_{N}$, for nitrogen-limited growth) rather than to its external concentration.

$$
\mu=\bar{\mu}_{\max } \cdot\left(1-\frac{q_{N, 0}}{q_{N}}\right)
$$

In Eq. (2), $\bar{\mu}_{\max }$ is the hypothetical maximum growth rate and $q_{N, 0}$ is the minimum nitrogen quota required for growth (Droop, 1968). This simple yet effective model has been used successfully to predict microalgae growth with additional terms accounting for multiple-nutrient limitation or the self-shading effects observed at high cell densities (Adesanya et al., 2014; Bernard, 2011; Bougaran et al., 2010; Mairet et al., 2011b; Packer et al., 2011). Microalgal cells can be autotrophic, heterotrophic, or mixotrophic, but most kinetic models describe solely autotrophic or heterotrophic growth. Adesanya et al. (2014) described the kinetics of a mixotrophically growing culture by expressing the hypothetical maximum growth rate as the sum of the autotrophic and heterotrophic growth rates. This approach was adapted into the present model with the inclusion of 
weighting functions controlling the extent of each rate on overall growth. The specific growth rate was thus expressed as:

$$
\mu=\bar{\mu}_{M, \max }(A, I) \cdot\left(1-\frac{q_{N, 0}}{q_{N}}\right)
$$

234 Here $\bar{\mu}_{M, \max }(A, I)$ is the maximum specific growth rate under mixotrophic conditions, as 235 shown in Eq. (4), and is proportional to the sum of the heterotrophic and phototrophic rates, $\mu_{H}(A)$ and $\mu_{I}(I)$, respectively.

$$
\bar{\mu}_{M, \max }(A, I)=\mu_{\max } \cdot\left[w_{H} \cdot \mu_{H}(A)+w_{I} \cdot \mu_{I}(I)\right]
$$

237 In order to account for photoinhibition and substrate inhibition, $\mu_{H}(A)$ and $\mu_{I}(I)$ were 238 expressed as Andrews functions (Andrews, 1968) as shown in Eq. (5):

$$
\bar{\mu}_{M, \max }(A, I)=\mu_{\max } \cdot\left[w_{H} \cdot \frac{A}{A+K_{S, A}+A^{2} / k_{i, A}}+w_{I} \cdot \frac{I}{I+K_{S, I}+I^{2} / k_{i, I}}\right]
$$

239 Here, $K_{S, A}$ and $k_{i, A}$ are the acetate-associated saturation and inhibition constants for growth, whereas $K_{s, I}$ and $k_{i, I}$ are the light-associated saturation and inhibition constants, respectively. The weighting functions, $w_{H}$ and $w_{I}$, shown in Eq. (6), were defined in terms of the saturation constants in a similar fashion to those presented in Shuler and Kargi (1992):

$$
w_{H}=\frac{A / K_{S, A}}{A / K_{S, A}+I / K_{S, I}} ; \quad w_{I}=\frac{I / K_{S, I}}{A / K_{S, A}+I / K_{S, I}}
$$

Light distribution $(I)$ throughout the culture vessel was represented by the Beer-Lambert law shown in Eq. (7), where $I_{0}\left(\mu \mathrm{mol} \mathrm{m} \mathrm{s}^{-1}\right)$ is the incident light intensity, $\sigma$ is the light attenuation coefficient, and $z(\mathrm{~m})$ is the culture depth within the vessel. 


$$
I=I_{0} \cdot e^{-\sigma \cdot X \cdot Z}
$$

247

248

\subsection{Nitrogen uptake rate, $\rho_{N}$}

The expression for nitrogen uptake rate is a crucial element of the model, since nitrogen entering the cells is directly linked to the nitrogen quota, which regulates cell growth (Eq. (3)). The uptake rate of nitrogen, shown in Eq.(8), was expressed as Andrews-type kinetics to account for the growth inhibition of $C$. reinhardtii observed at high external nitrogen concentrations. Since analogous observations were made in the cultures subject to high acetate treatments, acetate inhibition was similarly considered.

$$
\rho_{N}=\bar{\rho}_{N, \max }\left(N_{o}, X\right) \cdot \frac{N}{N+K_{S, N}+N^{2} / k_{i, N}} \cdot \frac{A}{A+K_{S, A: N}+A^{2} / k_{i, A: N}}
$$

Here, $K_{s, N}$ and $k_{i, N}$ are the nitrogen-associated saturation and inhibition constants for nitrogen uptake. Similarly, $K_{S, A: N}$ and $k_{i, A: N}$ are the acetate-associated saturation and inhibition constants for nitrogen uptake. The maximum nitrogen uptake rate, $\bar{\rho}_{N, \max }\left(N_{o}, X\right)$, depends on the initial nitrogen concentration $\left(N_{0}\right)$ under which cultures were grown and on the current biomass concentration $(X)$ :

$$
\bar{\rho}_{N, \max }\left(N_{0}, X\right)=\rho_{N, \max } \cdot \frac{N_{o}{ }^{n}}{N_{o}{ }^{n}+K_{*}{ }^{n}} \cdot e^{-\phi_{N} \cdot X}
$$

The maximum nitrogen uptake rate was built under the concept of "luxury consumption", used to describe the abrupt uptake of a nutrient from the cultivation medium (Droop, 1983). $K *$ is a saturation constant, $n$ is a shape-controlling parameter, and $\phi$ is a regulation coefficient. A detailed explanation is included in section 4.2.

\subsection{Rates of formation of cellular compartments $\left(R_{1}, R_{2}, R_{3}, R_{4}\right)$.}


The synthesis rates for starch and lipids, $R_{1}$ and $R_{3}$, respectively, were assumed to be

265

266

267

$$
\begin{aligned}
& R_{1}=r_{1} \cdot \frac{N_{i}^{n_{s}}}{N_{i}^{n_{s}}+K_{S, S}^{n_{S}}+\left(N_{i}^{2} / k_{i, S}\right)^{n_{s}}} \cdot \frac{k_{1}}{k_{1}+N / N_{o}} \cdot\left[1+\frac{1}{\mu} \cdot e^{\phi_{S} \cdot A_{\text {int }}}\right] \cdot \mu \cdot x^{*} \\
& R_{3}=r_{3} \cdot \frac{N_{i}^{n_{L}}}{N_{i}^{n_{L}}+K_{S, L}^{n_{L}}+\left(N_{i}^{2} / k_{i, L}\right)^{n_{L}}} \cdot \frac{k_{2}}{k_{2}+N / N_{0}} \cdot\left[1+\frac{1}{\mu} \cdot e^{\phi_{L} \cdot A_{i n t}}\right] \cdot \mu \cdot x^{*}
\end{aligned}
$$

273 Here, $K_{s, S}$ and $K_{s, L}$ are saturation constants, $k_{i, S}$ and $k_{i, L}$ are inhibition constants, $n_{s}$ and $n_{L}$ are shape-controlling exponents, $\Phi_{S}$ and $\Phi_{L}$ are regulation coefficients, and $k_{1}$ and $k_{2}$ regulate synthesis rates with respect to nitrogen consumption. Active biomass formation was also linked with starch and lipid degradation $\left(R_{2}\right.$ and $\left.R_{4}\right)$. These rates, shown in Eq. (12), were defined as functions of the nitrogen quota since cellular components such as proteins or nucleic acids, depend on nitrogen availability.

$$
R_{2}=\frac{r_{2}}{q_{N}} \cdot X ; R_{4}=\frac{r_{4}}{q_{N}} \cdot X
$$

\subsection{Time-dependent kinetic expressions.}


The dynamics for active biomass $\left(x^{*}\right)$, starch $(S)$, lipids $(L)$, and total biomass $(X)$, were obtained from the corresponding mass conservation equations as follows:

$$
\begin{gathered}
\frac{d x^{*}}{d t}=\mu \cdot X+R_{2}+R_{4}-\left(R_{1}+R_{3}\right) \\
\frac{d S}{d t}=R_{1}-R_{2} \\
\frac{d L}{d t}=R_{3}-R_{4}
\end{gathered}
$$

282 Total biomass (i.e. $X=x^{*}+S+L$ ) conservation simplifies thus to:

$$
\frac{d X}{d t}=\mu \cdot X
$$

283 Acetate consumption was expressed by means of the acetate to biomass yield

284 coefficient, $Y_{X / A}$, multiplied by a time-varying fraction accounting for the carbon used

285 heterotrophically:

$$
\frac{d A}{d t}=-\frac{1}{Y_{X / A}} \cdot \frac{\mu_{H}(A)}{\mu_{H}(A)+\mu_{I}(I)} \cdot \frac{d X}{d t}
$$

286 By considering that the removal of $\mathrm{H}^{+}$ions from the medium is a direct consequence of 287 microalgal growth (e.g. acetate consumption), $\mathrm{pH}$ evolution was represented as:

$$
\frac{d H}{d t}=K_{H} \cdot \frac{d x^{*}}{d t}
$$

Here, $K_{H}$ is a $\mathrm{pH}$ coefficient. The rate of nitrogen consumption was expressed as: 


$$
\frac{d N}{d t}=-\rho_{N} \cdot X
$$

289 Differentiation of the nitrogen quota with respect to time yields:

$$
\frac{d q_{N}}{d t}=\frac{d\left(N_{i} / X\right)}{d t}=\frac{\frac{d N_{i}}{d t} \cdot X-N_{i} \cdot \frac{d X}{d t}}{X^{2}}=\frac{1}{X} \cdot \frac{d N_{i}}{d t}-\frac{N_{i}}{X} \cdot\left(\frac{1}{x} \cdot \frac{d X}{d t}\right)
$$

where $N_{i}$ is the internal nitrogen concentration, and its accumulation rate is given by:

$$
\frac{d N_{i}}{d t}=-\frac{d N}{d t}=\rho_{N} \cdot X
$$

291 By substituting Eq. (16) and Eq. (21) in Eq. (20), the time-dependent equation for the 292 nitrogen quota simplifies thus to:

$$
\frac{d q_{N}}{d t}=\rho_{N}-\mu \cdot q_{N}
$$

\subsection{Parameter estimation.}

294

The proposed model, given by Eq.(13) - Eq.(19) and Eq.(22), consists of 8 state variables and 31 kinetic parameters (Table 1). Sensitivity analysis was carried out by estimating sensitivities (gradients of each state variable with respect to each of the parameters) numerically using central finite differences for a $10 \%$ change in each parameter. The results can be found in the Supplementary material. We noticed that sensitivities above value of 0.02 denoted that the corresponding variable was sensitive to changes in the parameter. Through this sensitivity analysis, 4 parameters were deemed insensitive, $\sigma, K_{s, I}, K_{S, S}$ and $\Phi_{L .} K_{S, S}$ and $\Phi_{\mathrm{L}}$ were neglected from the final model as it was noticed that setting them to zero did not affect results. $K_{s, I}$ was set to 1.4 as in 

become:

$$
\begin{gathered}
R_{1}=r_{1} \cdot \frac{N_{i}^{n_{S}}}{N_{i}^{n_{s}}+\left(N_{i}^{2} / k_{i, S}\right)^{n_{s}}} \cdot \frac{k_{1}}{k_{1}+N / N_{o}} \cdot\left[1+\frac{1}{\mu} \cdot e^{\phi_{S} \cdot A_{i n t}}\right] \cdot \mu \cdot x^{*} \\
R_{3}=r_{3} \cdot \frac{N_{i}^{n_{L}}}{N_{i}^{n_{L}}+K_{S, L}^{n_{L}}+\left(N_{i}^{2} / k_{i, L}\right)^{n_{L}}} \cdot \frac{k_{2}}{k_{2}+N / N_{0}} \cdot[\mu+1] \cdot x^{*}
\end{gathered}
$$
The value of each kinetic parameter was restricted to specified bounds according to data

Estimation of the remaining 27 kinetic parameters was carried out by minimizing an objective function defined as the sum of the squared relative error between the model predictions and the experimental data, as shown in (Vlysidis et al., 2011):

$$
\min G(P)=\sum_{h=1}^{n h} \sum_{i=1}^{n i} \sum_{k=1}^{n k}\left(\frac{Z_{h i k}^{\text {Pred }}(P)-Z_{h i k}^{\text {Exp }}}{Z_{h i k}^{E x p}}\right)^{2}
$$

where $G(P)$ is the objective function dependent on a vector $P$ containing all kinetic parameters and $Z$ is a vector containing all state variables, $n k$ is the number of experimental datasets used for parameter fitting, $n i$ is the number of state variables $(n i=$ $8)$, and $n h$ is the number of data points in time $(n h=7)$. Minimization of the objective function was performed by first employing Simulated Annealing (SA), a stochastic optimisation algorithm which is capable of approximating the solution set around a global minimum. Then, using the solution obtained by SA as initial guess, a refined and final solution set of parameters were computed by using Successive Quadratic Programming (SQP) (Vlysidis et al., 2011). Both techniques were coded in-house and implemented in MatLab ${ }^{\circledR}$ found in literature (for those cases in which available data existed) or relevant 
experimental analysis. Initial values for the model ODEs were equal to those implemented in each of the five experimental datasets used for parameter fitting $(n k=$ 5): TAP, N--, A++, High N, and High A-N. These datasets were selected so as to cover scenarios representative of the cultivation stage under both low and high concentrations of nitrogen and acetate. The remaining datasets ( $\mathrm{N}-, \mathrm{A}+$, and $\left.\mathrm{A}^{\prime}-\mathrm{N}^{\prime}\right)$ were used for model validation.

\section{Results and discussion.}

\subsection{Effect of nitrogen and acetate in biomass, starch, and lipid formation.}

All of the cultures analysed under the conditions established in section 2.2 reached early stationary phase after $150 \mathrm{~h}$, but cultures were allowed to grow for a further period of $48 \mathrm{~h}$ to ensure they had all reached stationary phase and were accumulating carbon storage products. Experimental results for biomass growth as well as for starch and lipid formation are shown in Figure 7, which are representative of the cultures during the stationary stage $(192 \mathrm{~h})$.

Results showed (Figure 7.b) that the cellular contents of starch and lipids increased significantly ( $p<0.0001$, two-way ANOVA) as the initial nitrogen concentration in the culture medium was reduced from $0.3824 \mathrm{gN} \mathrm{L}^{-1}$ (TAP), to both $0.3568 \mathrm{gN} \mathrm{L}^{-1}$ and $0.335 \mathrm{gN} \mathrm{L}^{-1}$. Specifically, starch concentration increased from $6 \%$ (at $N_{o}=0.3824 \mathrm{gN} \mathrm{L}^{-}$ ${ }^{1}$ ) to $17 \%$ (at $N_{o}=0.3350 \mathrm{gN} \mathrm{L}^{-1}$ ), whereas lipid increased from $14 \%$ to $21 \%$, respectively. This enhanced accumulation observed under nitrogen limitation is in agreement with previous analysis of C. reinhardtii (Bajhaiya et al., 2016) and with findings reported for other microalgae strains, such as Chlorella vulgaris P12 (Brányiková et al., 2010) or Tetraselmis subcordiformis (Yao et al., 2012). However, 
this increase is at the expense of biomass growth (Figure 7.a), which was observed to decrease significantly under nitrogen-limited conditions $(\mathrm{p}=0.0006$ between $0.3824 \mathrm{gN}$ $\mathrm{L}^{-1}$ and $0.335 \mathrm{gN} \mathrm{L}^{-1}$, two-way ANOVA). The magnitude of this negative trade-off in biomass growth ultimately controls starch and lipid formation in terms of volumetric yields, and should be considered in any nutrient-based cultivation strategy.

As per the ANOVA test, increases in starch and lipid contents in the culture grown at a high nitrogen concentration $\left(N_{o}=0.7426 \mathrm{gN} \mathrm{L}^{-1}\right)$ were not statistically significant with respect to the culture grown under standard TAP concentrations. However, biomass concentration decreased significantly ( $\mathrm{p}<0.0001$, two-way ANOVA), indicating that a high nitrogen concentration inhibited biomass growth. Nitrogen has been widely reported as a limiting nutrient suitable for increased accumulation of lipid (Cakmak et

Dragone et al., 2011). Nitrogen is a vital component of important biomolecules like proteins and DNA, and it is estimated to represent $7-20 \%$ of the cellular mass. When cells are exposed to a nitrogen depleted environment, the protein synthesis pathway is negatively affected, which results in the carbon fixation mechanism being instead redirected towards the production of carbohydrates or lipids (Juneja et al., 2013; Markou et al., 2012).

In addition, experimental results (Figure 7.c) showed that when compared to the culture grown in TAP $\left(A_{o}=0.42 \mathrm{gC} \mathrm{L}^{-1}\right)$, an increase in the initial acetate concentration had a significant effect on $C$. reinhardtii growth $\left(\mathrm{p}<0.0001\right.$ for $A_{o}=0.75 \mathrm{gC} \mathrm{L}^{-1}$ and $A_{o}=1.26$ $\mathrm{gC} \mathrm{L}^{-1} ; \mathrm{p}=0.0024$ for $\left.A_{o}=0.21 \mathrm{gC} \mathrm{L}^{-1}\right)$. Specifically, the biomass concentration $(192 \mathrm{~h})$ rose from $0.25 \mathrm{gC} \mathrm{L}^{-1}$ to $0.41 \mathrm{gC} \mathrm{L}^{-1}$ as the initial acetate concentration increased from $A_{o}=0.21 \mathrm{gC} \mathrm{L}^{-1}$ to $A_{o}=1.26 \mathrm{gC} \mathrm{L}^{-1}$. The presence of an additional organic carbon source 
367 (such as acetate) has been shown to: i) boost microalgal biomass growth (Chapman et 368 al., 2015), and ii) increase starch and lipid accumulation, caused possibly by either the 369 greater cell sizes of acetate-enhanced cultures (Goodson et al., 2011) or the larger availability of the carbon pool which shifts or lengthens the biosynthetic pathways (Fan et al., 2012; Goodenough et al., 2014). Although the cellular contents of the storage molecules increased slightly as a result of acetate addition (Figure 7.d), the extent of this accumulation was less noticeable $\left(\mathrm{p}>0.05\right.$, between $0.42 \mathrm{gC} \mathrm{L}^{-1}$ and all carbon treatments) than the nitrogen-driven accumulation.

Similar to nitrogen-limited growth observations, biomass concentration decreased at a high acetate concentration of $A_{o}=2.52 \mathrm{gC} \mathrm{L}^{-1}$. The combined inhibitory effects posed by high nitrogen and acetate concentrations were further verified experimentally in the HIGH A-N culture $\left(A_{o}=2.52 \mathrm{gC} \mathrm{L}^{-1}\right.$ and $\left.N_{o}=0.7430 \mathrm{gN} \mathrm{L}^{-1}\right)$, which attained a biomass concentration of $0.21 \mathrm{gC} \mathrm{L}^{-1}$ (Figure 7.e). Thus, the expected increase in costs for such a high-nutrient strategy, coupled with the growth inhibition, undermines its potential use for C. reinhardtii cultivation.

\subsection{Predictive performance of the kinetic model.}

The microalgae-based model developed in this work consists of 8 ODEs and 31 kinetic parameters. The estimated values of each kinetic parameter computed by the methodology described in Section 3.5 are presented in Table 1, which also provides reference values available in the open literature. The resulting concentration profiles of each state variable, as predicted by the model, are shown in Figure 8 against their corresponding experimental values. The model was capable of predicting accurately all 
experimental values in both datasets that were used in the fitting process (e.g. TAP and

N-) and datasets obtained at different conditions (e.g. A++ and N'-A') for validation.

Additional details are available as Supplementary Information.

393

394

The model was able to compute accurate dynamic concentration profiles for all species involved under different nitrogen and carbon concentration regimes (Figure 8), including total biomass, $X$, indicating that the Droop-based expression used for the specific growth rate (Eq.(3)) can adequately describe microalgal growth dynamics under nitrogen-limited mixotrophic conditions. Although both the classic Monod and Droop's model have been widely used to model microalgal growth, an added advantage of the Droop's model is its dependence on internal nutrient availability, which allows to capture the observed ability of microalgae to grow even after complete exhaustion of a limiting nutrient (Lee et al., 2015).

Small disagreements between predictions and experimental data can be seen for $\mathrm{pH}$ nitrogen dynamics (Figure 8.b, Figure 8.h). The variation in $\mathrm{pH}$ predictions might be the result of using a rather simple expression (Eq.(18)) that does not take into account the formation of other organic acids produced in small quantities by $C$. reinhardtii, such as formic acid or glycolic acid (Bekirogullari et al., 2017). Another potential cause for these disagreements could be related to the presence of tris-base in the cultivation medium. Tris-base acts as a biochemical buffer, but its concentration profile is not predicted by the kinetic model. Instead, tris-base is only implicitly included within nitrogen dynamics due to its high contribution to total nitrogen concentration: almost $70 \%$ of the total nitrogen present in standard TAP medium originates from tris-base. The latter might also explain the slight discrepancies observed between the predicted and experimentally obtained dynamics of nitrogen uptake. However, rather than 
incorporating individual uptake expressions for each nitrogen source, which would increase complexity and computational time, the kinetic model was built with one single expression for nitrogen uptake (Eq. (8)).

Models in which microalgal growth is limited by an internal nutrient pool generally assume Michaelis-Menten (MM) uptake kinetics, as in Droop's original approach (Droop, 1983). Under this assumption, nutrient uptake is assumed to be dependent on a single enzyme system that controls the uptake of extracellular substrates (Shuler and Kargi, 1992). In the current model, however, Andrews-type kinetics were employed to effectively predict $C$. reinhardtii's growth inhibition at high nitrogen concentrations. Inhibited uptake dynamics then cause less nitrogen to enter the cells, which translates into smaller nitrogen quota and, consequently, decreased growth.

Although MM-type kinetics have been successfully implemented in microalgaeoriented models (Adesanya et al., 2014; Mairet et al., 2011b; Packer et al., 2011), it was model) might suffer from the same weakness. This flaw is potentially a result of treating the maximum uptake rate, $\rho_{N, \operatorname{Max}}$, as a constant rather than as a dynamic variable (Morel, 1987), preventing an organism's uptake kinetics to respond to environmental changes (Bonachela et al., 2011). In microalgae, an abrupt increase in nutrient availability might lead to the phenomenon of luxury consumption, which refers to the sudden drop (uptake) of a nutrient from the surrounding medium (Droop, 1983). This phenomenon was observed in all our lab-scale experiments, where nitrogen concentration decreased 
luxury consumption was expected to be dependent on the current cell density and the nutrient concentration of the fresh medium (Droop, 1983), the maximum uptake rate (Eq. (9)) was expressed as a decreasing function of biomass (Zhang et al., 2008) coupled with a Monod-like function of the initial nitrogen concentration.

Moreover, the model was able to predict the higher nitrogen consumption yields observed in those cultures grown under high acetate concentrations. From the datasets shown in Figure 8.b, for example, it was estimated that the culture grown in $A_{o}=1.26$ $\mathrm{gC} \mathrm{L}^{-1}(\mathrm{~A}++)$ consumed about $93 \%$ of the total nitrogen supplied, whereas the culture grown in $A_{o}=0.42 \mathrm{gC} \mathrm{L}^{-1}$ (TAP) consumed 77\% (both cultures grown in $N_{o}=0.3824 \mathrm{gN}$ $\left.\mathrm{L}^{-1}\right)$. This is because cells require a large supply of nitrogen to compensate for acetateenhanced growth rates, as observed in this work and that of Chapman et al. (2015), where $C$. reinhardtii cells showed a higher growth rate under mixotrophic rather than phototrophic conditions.

As observed in Figure 8.e, Figure 8.f, the proposed model was also able to predict adequately the simultaneous concentration profile of starch and lipids under a wide range of initial nitrogen and acetate concentrations. The high predictive behaviour shown by the model proposed in this study, particularly for starch and lipid formation during nitrogen-limited mixotrophic growth conditions, confirms its potential as a robust tool in the development of optimal nutrient-based microalgal cultivation strategies. An optimisation study was thus undertaken and is presented next.

\subsection{Optimal nutrient-based strategies for starch and lipid formation.}

In order to profit from the accurate predictions obtained, the model was subsequently used to establish the optimal initial conditions to attain maximum concentrations of the 
two valuable biofuel feedstocks: starch and lipids. This procedure was carried out by identifying the maximum in a contour plot of each variable, as computed by the validated model, at a time equivalent to the point of highest storage molecule formation ( $t=192 \mathrm{~h}$ ). The resulting contour plots can be seen in Figure 9.

465

The maxima of the contour plots shown in Figure 9.b and Figure 9.c allow to identify optimal nitrogen and acetate concentrations that maximise lipid (lipid-enhanced scenario) or starch (starch-enhanced scenario). These optimal sets are: i) $\mathrm{OPT}_{\text {Starch }}=$ ( $A_{o}=1.06 \mathrm{gC} \mathrm{L}^{-1}$ and $N_{o}=0.336 \mathrm{gN} \mathrm{L}^{-1}$ ) for the starch-enhanced scenario, producing a starch concentration of $0.065 \mathrm{gC} \mathrm{L}^{-1}$ with corresponding lipid concentration of $0.069 \mathrm{gC}$ $\mathrm{L}^{-1}$; and ii) $\mathrm{OPT}_{\text {Lipids }}=\left(A_{o}=1.15 \mathrm{gC} \mathrm{L}^{-1}\right.$ and $\left.N_{o}=0.378 \mathrm{gN} \mathrm{L}^{-1}\right)$ for the lipid-enhanced scenario producing a lipid concentrations of $0.08 \mathrm{gC} \mathrm{L}^{-1}$ with corresponding starch concentration of $0.043 \mathrm{gC} \mathrm{L}^{-1}$, respectively.

When compared to the base case, TAP $=\left(A_{o}=0.42 \mathrm{gC} \mathrm{L}^{-1}\right.$ and $\left.N_{o}=0.3824 \mathrm{gN} \mathrm{L}^{-1}\right)$, which predicts a starch concentration of $0.018 \mathrm{gC} \mathrm{L}^{-1}$ and a lipid concentration of 0.048 $\mathrm{gC} \mathrm{L}^{-1}$, the starch-enhanced scenario accounts for a drastic increase in starch of $261 \%$ (and corresponding 44\% increase in lipids), whereas the lipid-enhanced scenario accounts for an increase in lipids of 66\% (and 139\% increase in starch). In each optimised case both starch and lipid concentrations are maximised with respect to the base case, due in part by the acetate boost (enhanced mixotrophic conditions), which was previously shown to increase biomass growth. Indeed, the model predicted that the highest microalgal concentration (Figure 9.a) could be achieved at $A_{o}=1.1 \mathrm{gC} \mathrm{L}^{-1}$ and $N_{o}=0.415 \mathrm{gN} \mathrm{L}^{-1}$. 
The starch and lipid enhanced-scenarios were further validated experimentally by growing two additional microalgal cultures under the initial concentrations $\mathrm{OPT}_{\text {Starch }}$ and $\mathrm{OPT}_{\mathrm{Lipids}}$, respectively. Predicted concentration profiles and data obtained experimentally from these enhanced scenarios are presented in Figure 10. For comparison, microalgal dynamics obtained by standard TAP concentrations are also plotted. As observed, the model performance proved once more its ability to capture adequately the trade-off between starch and lipid formation under nitrogen-limited mixotrophic growth. It could be argued that the magnitude of the predicted increases in biomass, starch, and lipids would not justify the required increase in acetate inputs. The good predictive performance of the model, however, allows carrying out alternate optimization strategies in which other factors are taken into account as per cultivation requirements (e.g. productivity, nutrient consumption yields, etc.). The outcome of such model-based optimised scenarios will undoubtedly aid in the development of microalgae as a biofuel feedstock by tackling challenges faced during the cultivation stage, such as reducing the nutrient-associated costs whilst simultaneously increasing starch and lipid productivities.

\section{Conclusions.}

A multi-parameter kinetic model was developed to predict nitrogen-limited mixotrophic microalgal growth coupled with simultaneous starch and lipid formation. All kinetic parameters were accurately computed by minimising the squared relative error between experimental values and model predictions. The predicted time-profiles of the model's state variables were then validated against additional experimental datasets obtained under different nutrient concentration regimes. Model-based optimised cultivation strategies, maximising starch (261\% increase with respect to base case) and lipid (66\% 
increase with respect to base case) production, were subsequently computed, and further experimentally validated.

\section{Acknowledgements}

Gonzalo M. Figueroa-Torres kindly acknowledges the Mexican National Council of Science and Technology (CONACyT) for its financial support.

\section{References.}

1. Adesanya, V.O., Davey, M.P., Scott, S.A., Smith, A.G., 2014. Kinetic modelling of growth and storage molecule production in microalgae under mixotrophic and autotrophic conditions. Bioresour. Technol. 157, 293-304.

2. Alonso, A.A., Molina, I., Theodoropoulos, C., 2014. Modeling Bacterial Population Growth from Stochastic Single-Cell Dynamics. Appl. Environ. Microbiol. 80, 5241-5253.

3. Andrews, J.F., 1968. A mathematical model for the continuous culture of microorganisms utilizing inhibitory substrates. Biotechnol. Bioeng. 10, 707-723.

4. Bajhaiya, A.K., Dean, A.P., Driver, T., Trivedi, D.K., Rattray, N.J.W., Allwood, J.W., Goodacre, R., Pittman, J.K., 2016. High-throughput metabolic screening of microalgae genetic variation in response to nutrient limitation. Metabolomics 12, 9.

5. Ball, S.G., Deschamps, P., 2009. The Chlamydomonas Sourcebook: Starch Metabolism, The Chlamydomonas Sourcebook. Elsevier.

6. Behrens, P.W., Bingham, S.E., Hoeksema, S.D., Cohoon, D.L., Cox, J.C., 1989. Studies on the incorporation of CO2 into starch by Chlorella vulgaris. J. Appl. Phycol. 1, 123-130.

7. Bekirogullari, M., Fragkopoulos, I.S., Pittman, J.K., Theodoropoulos, C., 2017. Production of lipid-based fuels and chemicals from microalgae: An integrated experimental and modelbased optimization study. Algal Res. 23, 78-87.

8. Bernard, O., 2011. Hurdles and challenges for modelling and control of microalgae for CO2 mitigation and biofuel production. J. Process Control 21, 1378-1389.

9. Bonachela, J.A., Raghib, M., Levin, S.A., 2011. Dynamic model of flexible phytoplankton nutrient uptake. Proc. Natl. Acad. Sci. 108, 20633-20638.

10. Bougaran, G., Bernard, O., Sciandra, A., 2010. Modeling continuous cultures of microalgae colimited by nitrogen and phosphorus. J. Theor. Biol. 265, 443-54.

11. Brányiková, I., Maršálková, B., Doucha, J., Brányik, T., Bišová, K., Zachleder, V., Vítová, 
M., 2010. Microalgae—novel highly efficient starch producers. Biotechnol. Bioeng. 108, 766-776.

12. Brennan, L., Owende, P., 2010. Biofuels from microalgae-A review of technologies for production, processing, and extractions of biofuels and co-products. Renew. Sustain. Energy Rev. 14, 557-577.

13. Cakmak, T., Angun, P., Demiray, Y.E., Ozkan, A.D., Elibol, Z., Tekinay, T., 2012. Differential effects of nitrogen and sulfur deprivation on growth and biodiesel feedstock production of Chlamydomonas reinhardtii. Biotechnol. Bioeng. 109, 1947-1957.

14. Chapman, S.P., Paget, C.M., Johnson, G.N., Schwartz, J.-M., 2015. Flux balance analysis reveals acetate metabolism modulates cyclic electron flow and alternative glycolytic pathways in Chlamydomonas reinhardtii. Front. Plant Sci. 6, 474.

15. Chen, F., Johns, M.R., 1994. Substrate inhibition of Chlamydomonas reinhardtii by acetate in heterotrophic culture. Process Biochem. 29, 245-252.

16. Choix, F.J., De-Bashan, L.E., Bashan, Y., 2012. Enhanced accumulation of starch and total carbohydrates in alginate-immobilized Chlorella spp. induced by Azospirillum brasilense: I. Autotrophic conditions. Enzyme Microb. Technol. 51, 294-9.

17. Dragone, G., Fernandes, B.D., Abreu, A.P., Vicente, A. a., Teixeira, J. a., 2011. Nutrient limitation as a strategy for increasing starch accumulation in microalgae. Appl. Energy 88, $3331-3335$.

18. Droop, M.R., 1983. 25 Years of Algal Growth Kinetics A Personal View. Bot. Mar. 26, 99.

19. Droop, M.R., 1968. Vitamin B12 and Marine Ecology. IV. The Kinetics of Uptake, Growth and Inhibition in Monochrysis Lutheri. J. Mar. Biol. Assoc. United Kingdom 48, 689-733.

20. Eriksen, N., Riisgård, F., Gunther, W., Lønsmann Iversen, J., 2007. On-line estimation of $\mathrm{O} 2$ production, $\mathrm{CO} 2$ uptake, and growth kinetics of microalgal cultures in a gas-tight photobioreactor. J. Appl. Phycol. 19, 161-174.

21. Escobar, J.C., Lora, E.S., Venturini, O.J., Yáñez, E.E., Castillo, E.F., Almazan, O., 2009. Biofuels: Environment, technology and food security. Renew. Sustain. Energy Rev. 13, $1275-1287$.

22. Fan, J., Yan, C., Andre, C., Shanklin, J., Schwender, J., Xu, C., 2012. Oil accumulation is controlled by carbon precursor supply for fatty acid synthesis in Chlamydomonas reinhardtii. Plant Cell Physiol. 53, 1380-1390.

23. Fouchard, S., Pruvost, J., Degrenne, B., Titica, M., Legrand, J., 2009. Kinetic modeling of light limitation and sulfur deprivation effects in the induction of hydrogen production with Chlamydomonas reinhardtii: Part I. Model development and parameter identification. Biotechnol. Bioeng. 102, 232-45. 
24. Goodenough, U., Blaby, I., Casero, D., Gallaher, S.D., Goodson, C., Johnson, S., Lee, J.-H., Merchant, S.S., Pellegrini, M., Roth, R., Rusch, J., Singh, M., Umen, J.G., Weiss, T.L.,

$$
\text { Wulan, T., 2014. The Path to Triacylglyceride Obesity in the sta6 Strain of Chlamydomonas }
$$
reinhardtii. Eukaryot. Cell 13, 591-613.

25. Goodson, C., Roth, R., Wang, Z.T., Goodenough, U., 2011. Structural Correlates of Cytoplasmic and Chloroplast Lipid Body Synthesis in Chlamydomonas reinhardtii and Stimulation of Lipid Body Production with Acetate Boost. Eukaryot. Cell 10, 1592-1606.

26. Harris, E.H., 1989. The Chlamydomonas sourcebook : a comprehensive guide to biology and laboratory use. Academic Press.

27. Johnson, X., Alric, J., 2013. Central Carbon Metabolism and Electron Transport in Chlamydomonas reinhardtii: Metabolic Constraints for Carbon Partitioning between Oil and Starch. Eukaryot. Cell 12, 776-793.

28. Juneja, A., Ceballos, R., Murthy, G., 2013. Effects of Environmental Factors and Nutrient Availability on the Biochemical Composition of Algae for Biofuels Production: A Review. Energies 6, 4607-4638.

29. Kumar, A., Guria, C., Chitres, G., Chakraborty, A., Pathak, A.K., 2016. Modelling of microalgal growth and lipid production in Dunaliella tertiolecta using nitrogen-phosphoruspotassium fertilizer medium in sintered disk chromatographic glass bubble column. Bioresour. Technol. 218, 1021-1036.

30. Lee, E., Jalalizadeh, M., Zhang, Q., 2015. Growth kinetic models for microalgae cultivation: A review. Algal Res. 12, 497-512.

31. Mairet, F., Bernard, O., Lacour, T., Sciandra, A., 2011a. Modelling microalgae growth in nitrogen limited photobiorector for estimating biomass , carbohydrate and neutral lipid, in: Preprints of the 18th IFAC World Congress. Milano (Italy), pp. 10591-10596.

32. Mairet, F., Bernard, O., Masci, P., Lacour, T., Sciandra, A., 2011b. Modelling neutral lipid production by the microalga Isochrysis aff. galbana under nitrogen limitation. Bioresour. Technol. 102, 142-9.

33. Markou, G., Angelidaki, I., Georgakakis, D., 2012. Microalgal carbohydrates: an overview of the factors influencing carbohydrates production, and of main bioconversion technologies for production of biofuels. Appl. Microbiol. Biotechnol. 96, 631-645.

34. Maršálková, B., Širmerová, M., Kuřec, M., Brányik, T., 2010. Microalgae Chlorella sp . as an Alternative Source of Fermentable Sugars. Chem. Eng. Trans. 21, 1279-1284.

35. McNichol, J., MacDougall, K.M., Melanson, J.E., McGinn, P.J., 2012. Suitability of Soxhlet Extraction to Quantify Microalgal Fatty Acids as Determined by Comparison with In Situ Transesterification. Lipids 47, 195-207. 
36. Moon, M., Kim, C.W., Park, W.-K., Yoo, G., Choi, Y.-E., Yang, J.-W., 2013. Mixotrophic growth with acetate or volatile fatty acids maximizes growth and lipid production in Chlamydomonas reinhardtii. Algal Res. 2, 352-357.

37. Morel, F.M.M., 1987. Kinetics of Nutrient Uptake and Growth in Phytoplankton. J. Phycol. $23,137-150$.

38. Packer, A., Li, Y., Andersen, T., Hu, Q., Kuang, Y., Sommerfeld, M., 2011. Growth and neutral lipid synthesis in green microalgae: a mathematical model. Bioresour. Technol. 102, $111-7$.

39. Rodolfi, L., Chini Zittelli, G., Bassi, N., Padovani, G., Biondi, N., Bonini, G., Tredici, M.R., 2009. Microalgae for oil: strain selection, induction of lipid synthesis and outdoor mass cultivation in a low-cost photobioreactor. Biotechnol. Bioeng. 102, 100-12.

40. Rügen, M., Bockmayr, A., Legrand, J., Cogne, G., 2012. Network reduction in metabolic pathway analysis: Elucidation of the key pathways involved in the photoautotrophic growth of the green alga Chlamydomonas reinhardtii. Metab. Eng. 14, 458-467.

41. Scaife, M.A., Merkx-Jacques, A., Woodhall, D.L., Armenta, R.E., 2015. Algal biofuels in Canada: Status and potential. Renew. Sustain. Energy Rev. 44, 620-642.

42. Shuler, M.L., Kargi, F., 1992. Bioprocess Engineering: Basic Concepts. Prentice Hall.

43. Turon, V., Baroukh, C., Trably, E., Latrille, E., Fouilland, E., Steyer, J.-P., 2014. Use of fermentative metabolites for heterotrophic microalgae growth: Yields and kinetics. Bioresour. Technol. 175C, 342-349.

44. Vlysidis, A., Binns, M., Webb, C., Theodoropoulos, C., 2011. Glycerol utilisation for the production of chemicals: Conversion to succinic acid, a combined experimental and computational study. Biochem. Eng. J. 58-59, 1-11.

45. Xin, L., Hu, H., Ke, G., Sun, Y., 2010. Effects of different nitrogen and phosphorus concentrations on the growth, nutrient uptake, and lipid accumulation of a freshwater microalga Scenedesmus sp. Bioresour. Technol. 101, 5494-500.

46. Yao, C., Ai, J., Cao, X., Xue, S., Zhang, W., 2012. Enhancing starch production of a marine green microalga Tetraselmis subcordiformis through nutrient limitation. Bioresour. Technol. 118, 438-44.

47. Zhang, K., Burns, I.G., Turner, M.K., 2008. Derivation of a Dynamic Model of the Kinetics of Nitrogen Uptake Throughout the Growth of Lettuce: Calibration and Validation. J. Plant Nutr. 31, 1440-1460.

48. Zhang, X.-W., Chen, F., Johns, M.R., 1999. Kinetic models for heterotrophic growth of Chlamydomonas reinhardtii in batch and fed-batch cultures. Process Biochem. 35, 385389. 
Figure 1. Schematic representation of the cellular compartments and flows used in the kinetic model. $X$, total biomass; $\mu$, specific growth rate; $\rho_{N}$, nitrogen uptake rate; $\mathrm{R}_{l}$, starch synthetic rate; $R_{3}$, lipid synthetic rate; $R_{2}$, starch degradation rate; $R_{4}$, lipid degradation rate.

649

Figure 2. Biomass production and corresponding distribution of carbon compartments at $t=192 \mathrm{~h}$ ( $8^{\text {th }}$ day of cultivation) for: a) b) N-dependent cultures (starting $A_{o}=0.42 \mathrm{gC}$ $\mathrm{L}^{-1}$ ), c) d) A-dependent cultures (starting $N_{o}=0.3824 \mathrm{gN} \mathrm{L}^{-1}$ ), and e) d) a high A-N culture. Treatments that do not share uppercase letters are significantly different $(\mathrm{p}<$ $0.05)$, as determined by two-way ANOVA.

655

Figure 3. Comparison between the predicted time-profile (lines) and experimental data

657 (points) for the cultures grown in: TAP $\left(A_{o}=0.42 \mathrm{gC} \mathrm{L}^{-1}, N_{o}=0.3824 \mathrm{gN} \mathrm{L}^{-1}\right), \mathrm{N}-$

$658 \quad\left(A_{o}=0.42 \mathrm{gC} \mathrm{L}^{-1}, N_{o}=0.356 \mathrm{gN} \mathrm{L}^{-1}\right), \mathrm{A}++\left(A_{o}=1.26 \mathrm{gC} \mathrm{L}^{-1}, N_{o}=0.3824 \mathrm{gN} \mathrm{L}^{-1}\right)$, and N'$659 \mathrm{~A}^{\prime}\left(A_{o}=1.16 \mathrm{gC} \mathrm{L}^{-1}, N_{o}=0.3151 \mathrm{gN} \mathrm{L}^{-1}\right)$. Fittig datasets: TAP and A++; Validating 660 datasets: N- and N'-A'. Data and standard deviation are the mean of 2 experimental 661 replicates.

662

Figure 4. Contour plots generated from model predictions for: a) biomass, b) starch, and c) lipid formation (at $t=190 \mathrm{~h}$ ) during $C$. reinhardtii cultivation.

Figure 5. Comparison between the predicted time-profile (lines) and experimental data (points) for the cultures grown in: TAP $\left(A_{o}=0.42 \mathrm{gC} \mathrm{L}^{-1}, N_{o}=0.3824 \mathrm{gN} \mathrm{L}^{-1}\right), \mathrm{OPT}_{\mathrm{Starch}}$ 669 $\left(A_{o}=1.06 \mathrm{gC} \mathrm{L}^{-1}\right.$ and $\left.N_{o}=0.336 \mathrm{gN} \mathrm{L}^{-1}\right)$, and $\mathrm{OPT}_{\text {Lipids }}\left(A_{o}=1.15 \mathrm{gC} \mathrm{L}^{-1}\right.$ and $N_{o}=0.378 \mathrm{gN}$ $\left.\mathrm{L}^{-1}\right)$. Data and standard deviation are the mean of 2 experimental replicates. 
Table 1. Kinetic parameters used in our proposed model.

\begin{tabular}{|c|c|c|c|c|c|}
\hline & Parameter description & Value & Units & Strain & Reference \\
\hline \multirow{3}{*}{$\mu_{\max }$} & \multirow{3}{*}{$\begin{array}{l}\text { Maximum specific growth } \\
\text { rate }\end{array}$} & 0.106 & & C. reinhardtii & This work \\
\hline & & 0.227 & $\mathrm{~h}^{-1}$ & C. reinhardtii & Fouchard et al., 2009 \\
\hline & & 0.084 & & C. reinhardtii & Chen and Johns, 1994 \\
\hline \multirow{3}{*}{$q_{N, 0}$} & \multirow{3}{*}{ Minimum nitrogen quota } & 0.876 & $\mathrm{gN} \mathrm{gC}^{-1}$ & C. reinhardtii & This work \\
\hline & & 0.25 & $\mathrm{gN} \mathrm{gC}^{-1}$ & I. galbana & Bernard, 2011 \\
\hline & & 0.0975 & 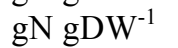 & C. vulgaris & Adesanya et al., 2014 \\
\hline \multirow{3}{*}{$K_{s, A}$} & \multirow{3}{*}{ Saturation constant, $A$} & 1.789 & $\mathrm{gC} \mathrm{L}^{-1}$ & C. reinhardti & This work \\
\hline & & $0.014^{a}$ & $\mathrm{gC} \mathrm{L}^{-1}$ & C. reinhardti & Chen and Johns, 1994 \\
\hline & & $1.04^{a}$ & $\mathrm{gC} \mathrm{L}^{-1}$ & C. reinhardti & Zhang et al., 1999 \\
\hline \multirow{3}{*}{$k_{i, A}$} & \multirow{3}{*}{ Inhibition constant, $A$} & 0.109 & $\mathrm{gC} \mathrm{L}^{-1}$ & C. reinhardti & This work \\
\hline & & $0.708^{a}$ & $\mathrm{gC} \mathrm{L}^{-1}$ & C. reinhardti & Chen and Johns, 1994 \\
\hline & & $0.042^{a}$ & $\mathrm{gC} \mathrm{L}^{-1}$ & C. reinhardti & Zhang et al., 1999 \\
\hline$K_{s, I}$ & Saturation constant, $I$ & 1.4 & $\mu_{\mathrm{mol}} \mathrm{m}^{-2} \mathrm{~s}^{-1}$ & I. galbana & Mairet et al., 2011a \\
\hline \multirow{2}{*}{$k_{i, I}$} & \multirow{2}{*}{ Inhibition constant, $I$} & 186.52 & $\mathrm{~m}^{-2} \mathrm{~s}^{-1}$ & C. reinhardti & This work \\
\hline & & 295.00 & $\mu_{\mathrm{mol}} \mathrm{m}^{-\mathrm{s}}$ & I. galbana & Mairet et al., 2011a \\
\hline \multirow{3}{*}{$Y_{X / A}$} & \multirow{3}{*}{ Yield coefficient } & 0.059 & ${\mathrm{gC} \mathrm{gC}^{-1}}^{-1}$ & C. reinhardti & This work \\
\hline & & 0.84 & ${\mathrm{gC} \mathrm{gC}^{-1}}^{-1}$ & C. sorokiniana & Turon et al., 2014 \\
\hline & & 0.50 & $\mathrm{~g} \mathrm{~g}^{-1}$ & C. reinhardtii & Chen and Johns, 1994 \\
\hline$\sigma$ & Light attenuation coefficient & 1 & $\mathrm{~L} \mathrm{gC}^{-1} \mathrm{~m}^{-1}$ & C. reinhardtii & This work \\
\hline$\rho_{N, \max }$ & Maximum $N$ uptake rate & 40.445 & $\mathrm{gN} \mathrm{gC}^{-1} \mathrm{~h}^{-1}$ & C. reinhardtii & This work \\
\hline$K_{*}$ & Saturation constant, $N_{o}$ & 0.3125 & $\mathrm{gN} \mathrm{L}^{-1}$ & C. reinhardtii & This work \\
\hline$n$ & Shape parameter & 18.183 & - & C. reinhardtii & This work \\
\hline$\Phi_{N}$ & Uptake regulation coefficient & 137.455 & $\mathrm{LgC}^{-1}$ & C. reinhardtii & This work \\
\hline$K_{s, N}$ & Uptake saturation constant, $N$ & 0.162 & $\mathrm{gN} \mathrm{L}^{-1}$ & C. reinhardtii & This work \\
\hline$k_{i, N}$ & Uptake inhibition constant, $N$ & 0.113 & $\mathrm{gN} \mathrm{L}^{-1}$ & C. reinhardtii & This work \\
\hline$K_{s, A: N}$ & Uptake saturation constant, $A$ & 1.004 & $\mathrm{gC} \mathrm{L}^{-1}$ & C. reinhardtii & This work \\
\hline$k_{i, A: N}$ & Uptake inhibition constant, $A$ & 1.098 & $\mathrm{gC} \mathrm{L}^{-1}$ & C. reinhardtii & This work \\
\hline$r_{1}$ & Rate of reaction, $R_{I}$ & 0.0420 & $\mathrm{gC} \mathrm{gC}^{-1}$ & C. reinhardtii & This work \\
\hline$r_{2}$ & Rate of reaction, $R_{2}$ & 0.1620 & $\mathrm{gN} \mathrm{gC}^{-1} \mathrm{~h}^{-1}$ & C. reinhardtii & This work \\
\hline$r_{3}$ & Rate of reaction, $R_{3}$ & 0.0041 & $\mathrm{gC} \mathrm{gC}^{-1}$ & C. reinhardtii & This work \\
\hline$r_{4}$ & Rate of reaction, $R_{4}$ & 0.0049 & $\mathrm{gN} \mathrm{gC}^{-1} \mathrm{~h}^{-1}$ & C. reinhardtii & This work \\
\hline$K_{S, S}$ & Saturation constant for $R_{I}$ & 0 & $\mathrm{gN} \mathrm{L}^{-1}$ & C. reinhardtii & This work \\
\hline$k_{i, S}$ & Inhibition constant for $R_{I}$ & 0.2079 & $\mathrm{gN} \mathrm{L}^{-1}$ & C. reinhardtii & This work \\
\hline$n_{S}$ & Shape parameter for $R_{I}$ & 3.6205 & - & C. reinhardtii & This work \\
\hline$k_{1}$ & Regulation constant for $R_{l}$ & 0.1771 & - & C. reinhardtii & This work \\
\hline$\Phi_{S}$ & Regulation coefficient for $R_{1}$ & 0.6675 & $\mathrm{LgC}^{-1}$ & C. reinhardtii & This work \\
\hline$K_{s, L}$ & Saturation constant for $R_{3}$ & 0.0227 & $\mathrm{gN} \mathrm{L}^{-1}$ & C. reinhardtii & This work \\
\hline$k_{i, L}$ & Inhibition constant for $R_{3}$ & 0.0861 & $\mathrm{gN} \mathrm{L}^{-1}$ & C. reinhardtii & This work \\
\hline$n_{L}$ & Shape parameter for $R_{3}$ & 1.8117 & - & C. reinhardtii & This work \\
\hline$k_{2}$ & Regulation constant for $R_{3}$ & 0.2135 & - & C. reinhardtii & This work \\
\hline$\Phi_{L}$ & Regulation coefficient for $R_{3}$ & 0 & $\mathrm{LgC}^{-1}$ & C. reinhardtii & This work \\
\hline$K_{H}$ & $\mathrm{pH}$ coefficient & 4.653 & $\mathrm{~L} \mathrm{gC}^{-1} \mathrm{~h}^{-1}$ & C. reinhardtii & This work \\
\hline
\end{tabular}




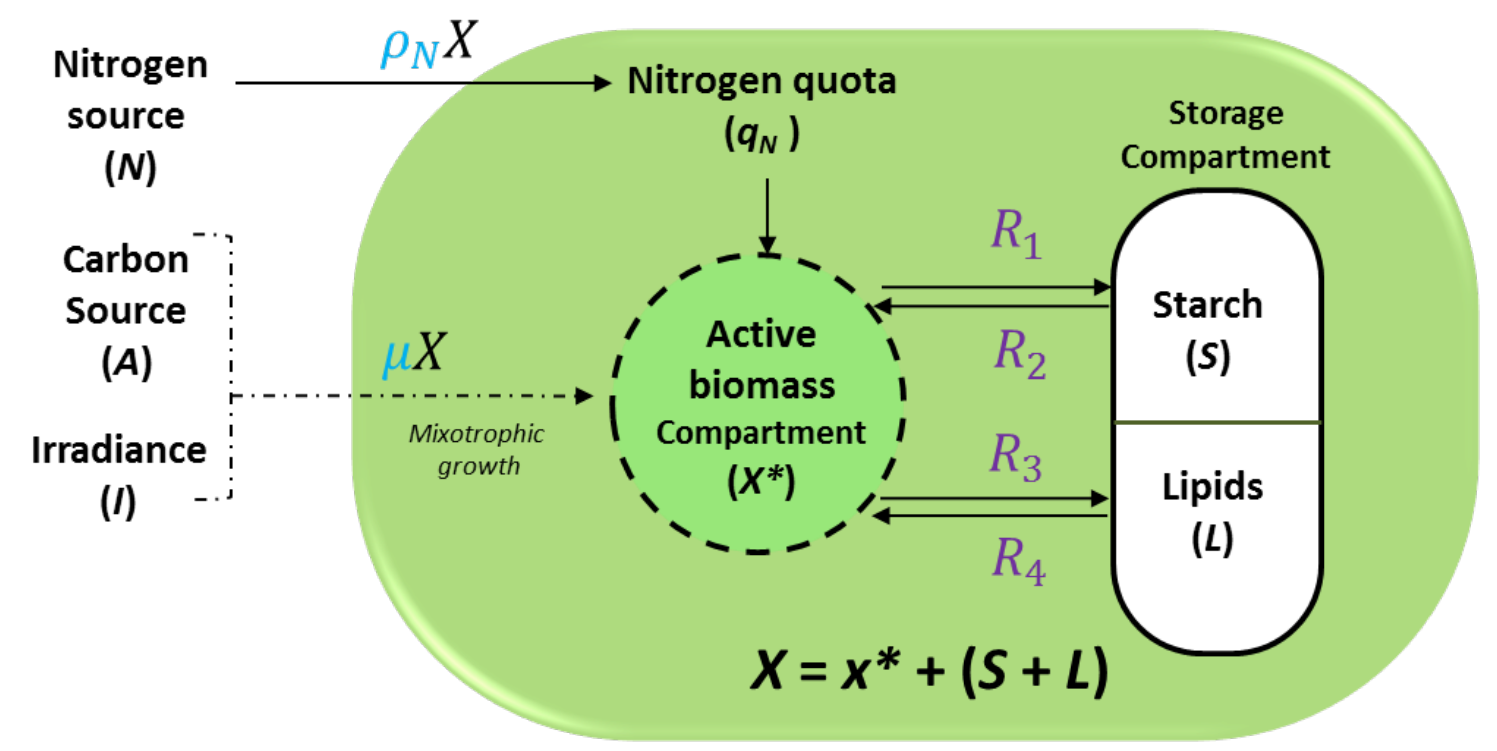



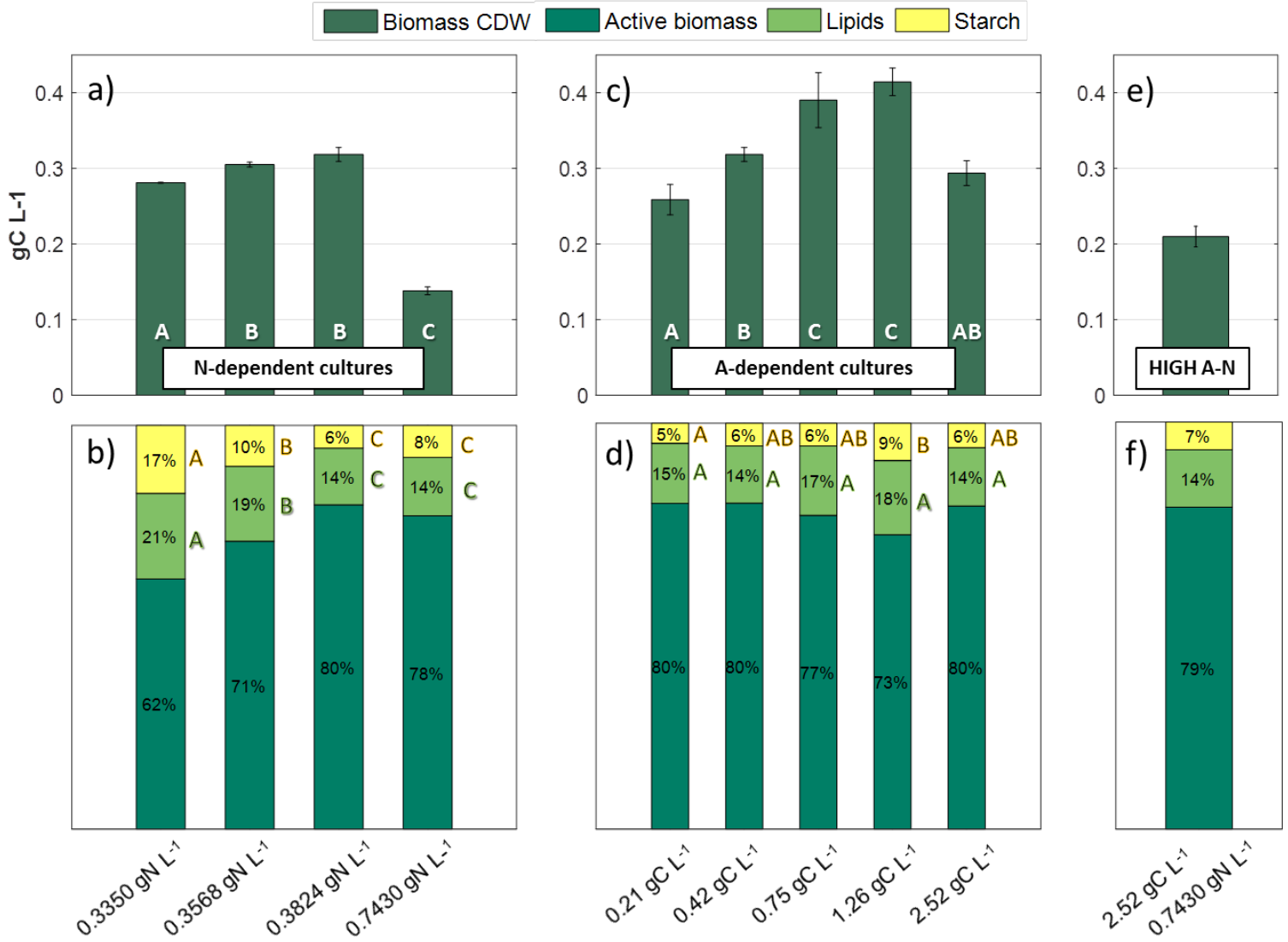

Figure 7.

688

689

690 

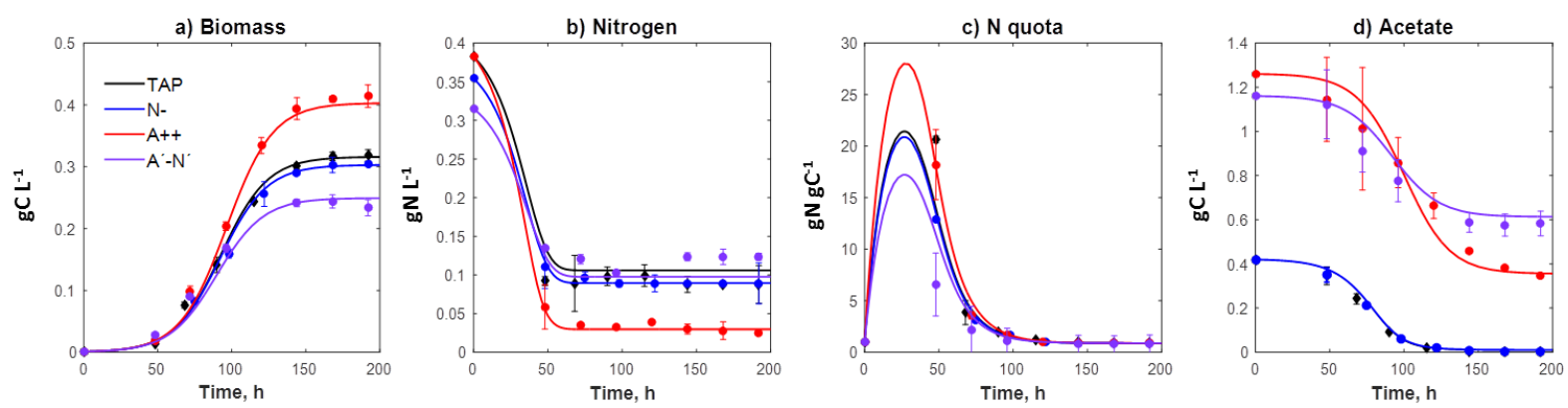

691
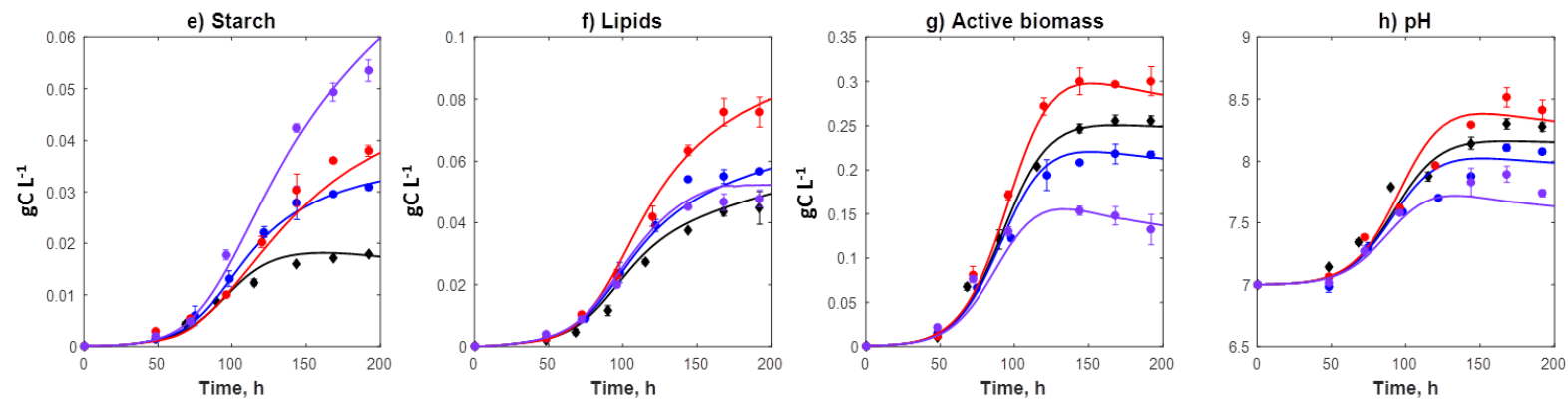

Figure 8.

693 


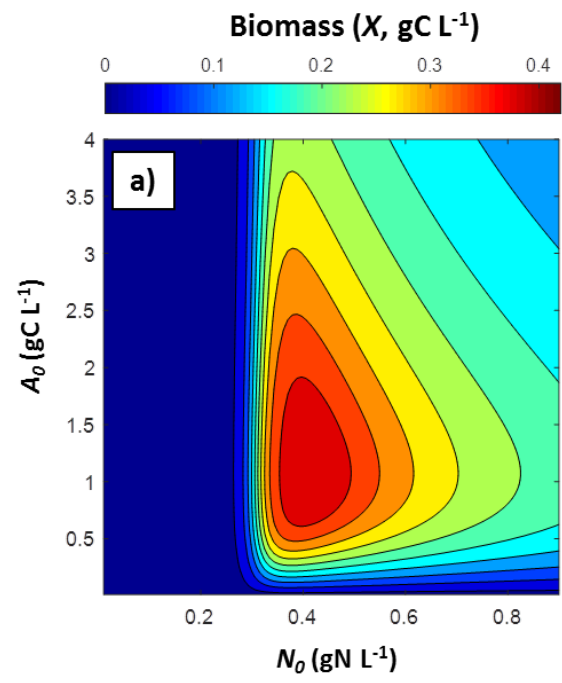

696

697
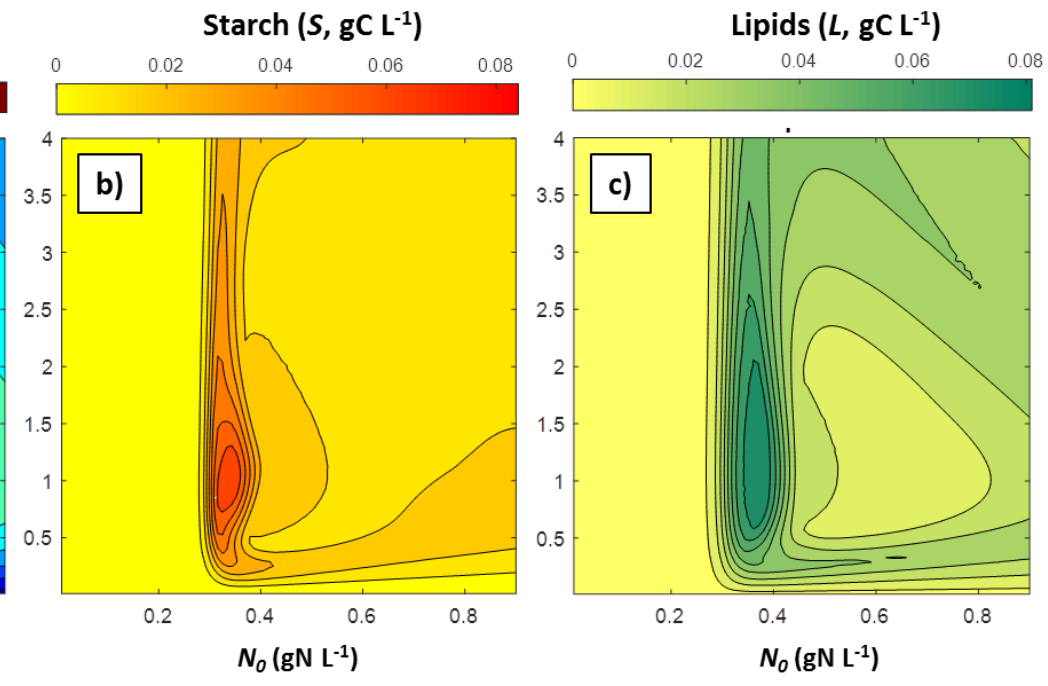

Figure 9. 

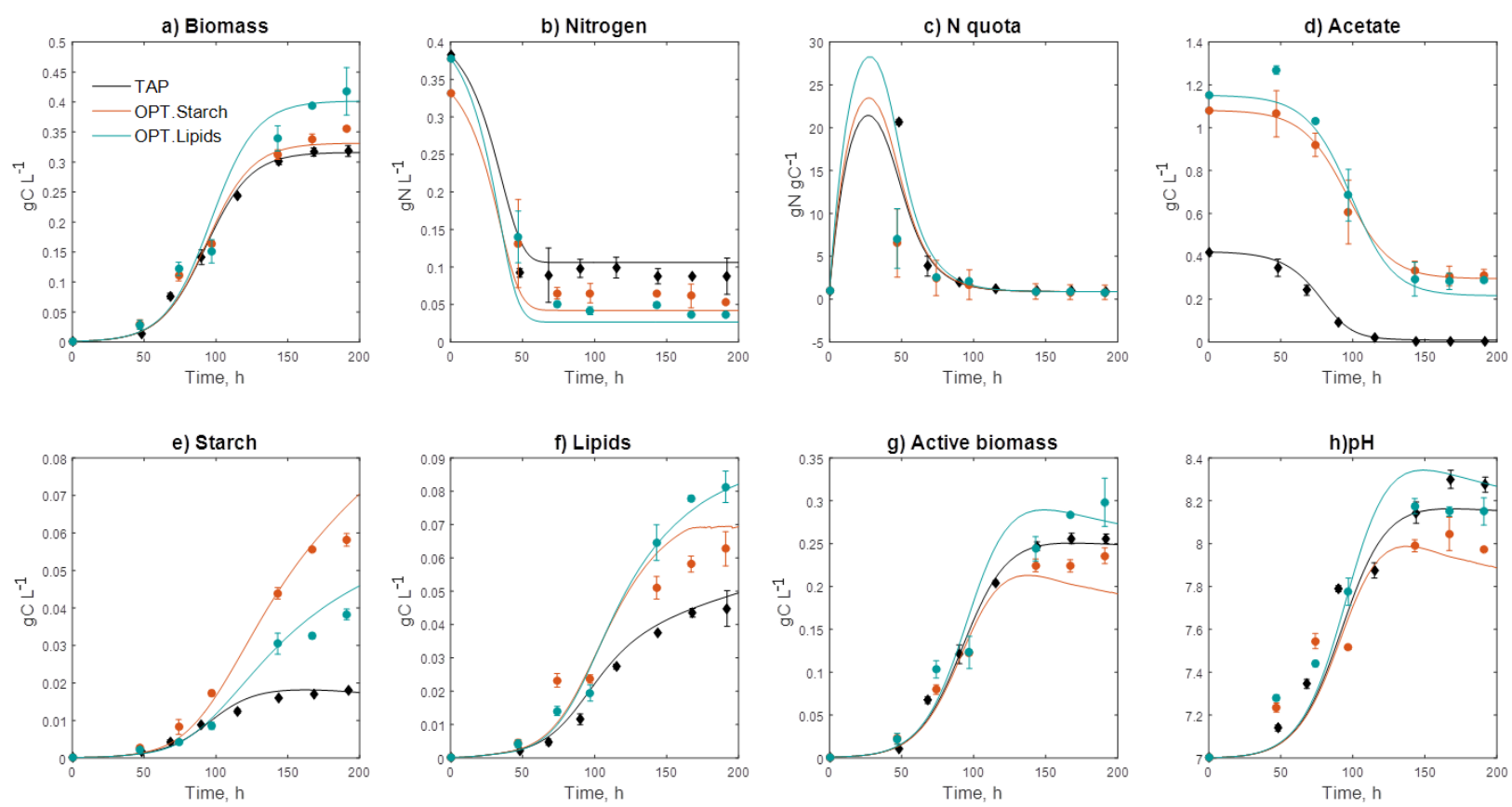

Figure 10. 